SHORT COMMUNICATION

\title{
Nonvolatile and Shape-Memorized Bacterial Cellulose Gels Swollen by Poly(ethylene glycol)
}

\author{
By Yukari Numata, ${ }^{1, *}$ Kazumi MURomoto,${ }^{1}$ Hidemitsu FuRUKaWA, ${ }^{2}$ \\ Jian Ping GONG, ${ }^{2}$ Kenji TAJIMA, ${ }^{3}$ and Masanobu MUNEKATA ${ }^{3}$
}

KEY WORDS: Biocompatibility / Cellulose / Gels / Stimuli-Sensitive Polymer / Transparency /

Bacterial cellulose (BC) provides a unique kind biopolymer gel, containing over 99 wt.- $\%$ water, produced by bacteria of genus Acetobacter. ${ }^{1}$ The BC gels have various useful properties such as softness, translucence, biocompatibility, and water retention ability..$^{2-5}$ This is why the BC gels receive much attention as potential soft materials for medicine, cosmetic, food, and etc. However, a large amount of water in the $\mathrm{BC}$ gels is quite easily squeezed out under small pressure and also easily dried in atmosphere. Thus, it is difficult to use the BC gels as engineering materials in practical applications. In order to overcome these limitations, many attempts were made to improve the mechanical strength of the $\mathrm{BC}$ gels. Recently, a breakthrough had come in gel science; synthetic tough hydrogels with anomalously high mechanical strength were developed by a double-network (DN) structure. ${ }^{6}$ Based on this technique, the $\mathrm{BC}$ gels with DN structure resulted higher mechanical strength than usual BC gels. $^{7}$ However, another problem is still unsettled about easy drying of BC gels in atmosphere; desiccation-tolerant, nonvolatile BC gels have been keenly sought.

Here, we have developed novel nonvolatile and thermo-responsive BC gels by introducing poly(ethylene glycol) (PEG) as nonvolatile solvents in BC gels instead of water solvent. It is known that PEG has good biocompatibility, nonvolatile, and water solubility, and shows various physical properties, depending on its molecular weight. Therefore, we tried to prepare different nonvolatile $\mathrm{BC}$ gels with different physical properties by changing the molecular weight $M_{\mathrm{w}}$ of PEG; the BC gels were swollen in three kinds of PEG melts, which are of $M_{\mathrm{w}} 200$ (named 'BC/PEG 200 gel'), 1000 (named 'BC/PEG 1000 gel'), and a mixture of 200 and 1000 (named 'BC/PEG 200-1000 gel'), where PEGs of $M_{\mathrm{w}} 200$ and 1000 are in liquid and solid states at room temperature, respectively. Also, we found the BC/PEG 200-1000 showed shape-memory property. In the present paper, we report these interesting and useful properties of the $\mathrm{BC} / \mathrm{PEG}$ gels.

\section{EXPERIMENTAL}

BC gels were biosynthesized by Acetobacter xylinum ATCC53582 in Hestrin and Schramm medium ${ }^{1}$ at $28^{\circ} \mathrm{C}$ for $4 \mathrm{~d}$ under the static condition. Subsequently, sample gels were purified by $1 \mathrm{wt} . \% \mathrm{NaOH}$ aqueous solution for deproteinization and washed with distilled water.

PEG 200 and PEG 1000 were purchased from Kishida Chemical Co., Ltd. PEG 200-1000 was obtained by mixing the same volume of PEG 200 and PEG 1000. Then, the purified BC gels were immersed in these aqueous solutions of PEG, and heated for $90 \mathrm{~min}$ in the air until water has removed almost completely. The surfaces of sample gels were washed with distilled water to remove an excess of PEG left on the BC gel surfaces. The gels were dried at $65^{\circ} \mathrm{C}$ for $1 \mathrm{~d}$ in the air on the glass plate.
In drying experiment, the sample gels were kept at $28^{\circ} \mathrm{C}$ in the air on the glass plate. The appearance of the gels was photographed by digital camera before and after the drying treatment. Measurements of water content ratios were performed with electric moisture gauge SHIMADZU MOC-120H. The PEG content ratio of the sample gels were determined by the weight ratio of the cellulose fiber and the evaporated water. DSC measurements were performed with SII SSC/5200 (SEIKO Ins.) in the presence of nitrogen. The sample gels were then heated from $10^{\circ} \mathrm{C}$ to $350^{\circ} \mathrm{C}$ at a rate of $10^{\circ} \mathrm{C} / \mathrm{min}$.

\section{RESULTS AND DISCUSSION}

Figure 1(a) demonstrates purified BC gel swollen in water. The emblem under the gel was visible despite its weak turbidity. The $\mathrm{BC}$ gel after drying treatment at $28^{\circ} \mathrm{C}$ for 2 weeks is demonstrated in Figure 1(b). After the drying treatment, the water in the $\mathrm{BC}$ gel evaporated almost completely and the $\mathrm{BC}$ gel became as film; the thickness decreased less than one-tenth thinner than before drying and the diameter also decreased approximately $10 \%$. On the other hand, Figure 1(c) demonstrates BC gel swollen in PEG 200. The transparency of the gels was significantly improved by introducing PEG as a solvent, in comparison with the BC gel swollen in water. Additionally, the gel kept both the thickness and good transparency during drying treatment at $28^{\circ} \mathrm{C}$ for 2 weeks. In this way, novel nonvolatile and highly transparent $\mathrm{BC} / \mathrm{PEG}$ gels have been successfully developed. The PEG molecules filled pores of $\mathrm{BC}$ network and were not covalently crosslinked to one another or BC surfaces. It implies that the PEG in the gels is squeezed by pressure. It should be improved by crosslinking in future.

Figure 2(a) demonstrates the temperature dependencies of nonvolatile $\mathrm{BC}$ gels swollen in the different molecular weight PEG. These BC/PEG gels have the small water content and the large PEG content, which was about 2 wt.- $\%$ and 98 wt.- $\%$, respectively. The BC/PEG 200 gel kept its good transparency independently of the temperature. The BC/PEG 2001000 gel was opaque and more inelastic than purified $\mathrm{BC}$ gel swollen in water at room temperature. However, the BC/PEG 200-1000 gel became transparent and elastic as the temperature increased above room temperature. The $\mathrm{BC} / \mathrm{PEG} 1000$ gel was more opaque at room temperature and less elastic than the BC/PEG 200-1000 gel at room temperature. After the temperature increased from room temperature, the BC/PEG 1000 gel remained in opaque and solid state at $28^{\circ} \mathrm{C}$. However, as the temperature increased further, it became elastic and well transparent at $40^{\circ} \mathrm{C}$. Then, as the temperature decreased, the BC/PEG 200-1000 and BC/PEG 1000 gels returned to the opaque and inelastic states, reversibly. It implies that the BC/PEG 200-1000 and BC/PEG 1000 gels have reversible thermoresponsibility. This reversibility comes from the solid-liquid transition of the PEG in the gels, which can be controlled by the molecular weight of the PEG.

\footnotetext{
${ }^{1}$ Department of Materials Chemistry, Asahikawa National College of Technology, Asahikawa 071-8142, Japan

${ }^{2}$ Department of Biological Sciences, Graduate School of Science, Hokkaido University, Sapporo 060-0810, Japan

${ }^{3}$ Division of Biotechnology and Macromolecular Chemistry, Graduate School of Engineering, Hokkaido University, Sapporo 060-8628, Japan

*To whom correspondence should be addressed (Tel: +81-166-55-8042, Fax: +81-166-55-8042, E-mail: numata@ asahikawa-nct.ac.jp).
} 
(a)

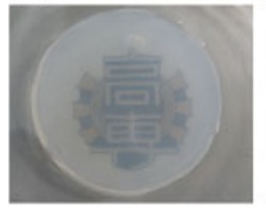

(b)

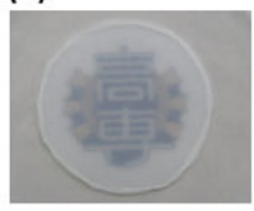

(c)

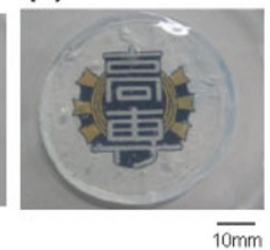

Figure 1. (a) Purified $B C$ gel swollen in water. (b) $B C$ gel after drying treatment at $28^{\circ} \mathrm{C}$ for 2 weeks. (c) Nonvolatile and transparent BC gel swollen in PEG after drying treatment at $28^{\circ} \mathrm{C}$ for 2 weeks.

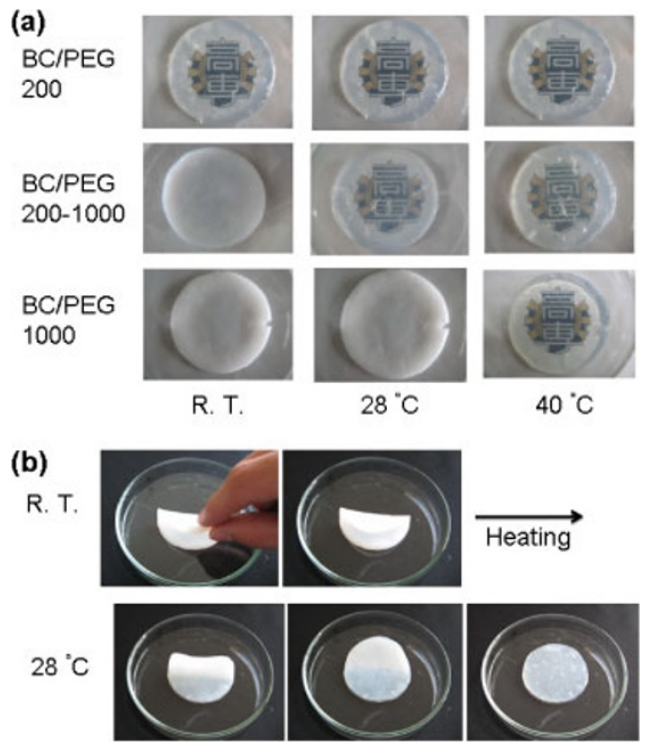

Figure 2. (a) Temperature dependence of nonvolatile $B C$ gels swollen in the different molecular weight $M_{\mathrm{w}}$ of PEG. (b) Shape-memory of BC/ PEG 200-1000. At room temperature, once the gel was folded, it kept a folded form. As the temperature increased toward $28^{\circ} \mathrm{C}$, the gel was soon restored to its original shape. After a few minutes more, the gel became completely transparent.

Figure 2(b) demonstrates the shape-memory property of BC/PEG 2001000. At room temperature, once the gel was folded, it kept its folded form. As the temperature increased, the gel started to become soft and transparent, and was soon restored to its original flat shape. A few minutes later, the gel became completely transparent. It is a somewhat interesting property of the BC/PEG gels, which can be applied to develop some thermo-responsive materials. It is expected that the shape-memory property of the BC/PEG gels depends on the network structure and mechanical properties of $\mathrm{BC}$ gels, as well as the molecular weight of PEG. We are studying further and will provide detailed report somewhere in future.

Figure 3 demonstrates the DSC results of the nonvolatile BC gels. The dashed curve indicates the endotherm of pure PEGs, and the solid curve indicates the endotherm of $\mathrm{BC}$ gels swollen in PEG. As demonstrated in Figure 3(a), in the BC/PEG 200-1000 and the PEG 200-1000, the sharp peak around $40^{\circ} \mathrm{C}$ in the PEG melt was observed. We considered that the (a)

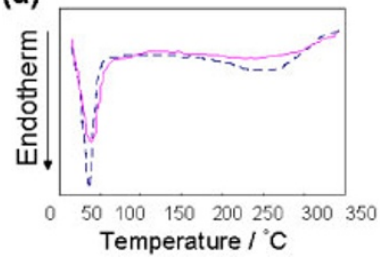

(b)

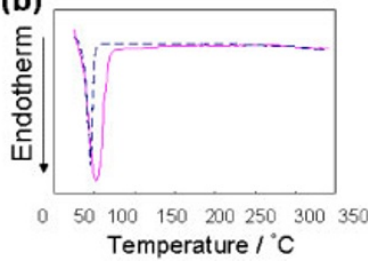

Figure 3. $D S C$ results of nonvolatile $B C$ gels swollen in (a) a mixture of PEG 200 and 1000, and (b) BC/PEG 1000. The dashed curve indicates the endotherm of pure PEGs, and the solid curve indicates the endotherm of $\mathrm{BC}$ gels swollen in PEG.

peak came from the melting process of the PEG 1000, which shifted to little higher temperature in the $\mathrm{BC} / \mathrm{PEG}$ gels due to the existence of PEG surrounding $\mathrm{BC}$ network. It implies that $\mathrm{BC}$ and PEG molecules weakly interact to suppress the melting process of PEG. As demonstrated in Figure 3(b), the peak of the melting process of PEG 1000 in the BC/PEG 1000 gel was observed at $51^{\circ} \mathrm{C}$. The shift of the melting point due to the gelation was larger than that observed in the case of BC/PEG 200-1000 gel. It implies that $\mathrm{BC}$ and PEG molecules interact more effectively. We consider that this interaction is important to control the thermo-responsibility and improve the mechanical properties of the BC/PEG gels.

In summary, we have succeeded in developing nonvolatile and thermoresponsive $\mathrm{BC} / \mathrm{PEG}$ gels. It is possible to control the transition temperature of these gels by altering the molecular weight $M_{\mathrm{w}}$ of PEG. Around the transition temperature, the gels change their transparency and elastic properties. Furthermore, the thermo-sensitive change in elastic properties brings the shape-memory nature of the BC/PEG gels. The DSC result indicated that it is possible to improve the shape-memory performance by controlling the interaction between the BC network and PEG. We believe that the function of the BC/PEG gels will be significantly improved by further studies and provide novel functional biocompatible soft materials, having powerful applicability to various fields, such as cosmetic, food, medicine and so on.

Received: February 10, 2009

Accepted: March 19, 2009

Published: May 13, 2009

\section{REFERENCES}

1. S. Hestrin and M. Schramm, Biochem. J., 58, 345 (1954).

2. T. Miyamoto, S. Takahashi, H. Ito, H. Inagaki, and Y. Noishiki, J. Biomed. Mater. Res., Part A, 23, 125 (1989).

3. A. Okiyama, M. Motoki, and S. Yamanaka, Food Hydrocolloids, 6, 493 (1993).

4. J. W. Lee, F. Deng, W. G. Yeomans, A. L. Allen, R. A. Gross, and D. L. Kaplan, Appl. Environ. Microbiol., 67, 3970 (2001).

5. D. Klemm, B. Heublein, H. P. Fink, and A. Bohn, Angew. Chem., Int. Ed., 44, 3358 (2005).

6. J. P. Gong, Y. Katsuyama, T. Kurokawa, and Y. Osada, Adv. Mater., 15, 1155 (2003).

7. A. Nakayama, A. Kakugo, J. P. Gong, Y. Osada, M. Takai, T. Erata, and S. Kawano, Adv. Funct. Mater., 14, 1124 (2004). 